\title{
Quantitative version of a Silverstein's result
}

\author{
Alexander E. Litvak Susanna Spektor
}

\begin{abstract}
We prove a quantitative version of a Silverstein's Theorem on a condition for convergence in probability of the norm of random matrix. More precisely, we show that for a random matrix whose entries are i.i.d. random variables, $w_{i, j}$, satisfying certain natural conditions, is not small with large probability.
\end{abstract}

Let $\left\{w_{i j}\right\}$ be i.i.d. random variables, identical copies of a certain random variable $w$. Conditions on $w$ (e.g. moments, tails) will be mentioned later. For each positive integer $n$ we consider a $p \times n$ matrix $W_{n}=\left(w_{i j}\right), i=1,2 \ldots, p, j=1,2 \ldots, n$. We study $\lambda_{\max }\left(\Gamma_{n}\right)$, the largest eigenvalue of the sample covariance matrix $\Gamma_{n}:=\frac{1}{n} W_{n} W_{n}^{T}$, of $n$ samples of a $p$-dimensional vector containing i.i.d. components, where $p$ and $n$ are large. In the present work we considered the case when $p \leq n$, otherwise everything will work with conjugate matrices.

It was proved in [1], see also [2], that with $p=p(n)$ satisfies $\frac{p}{n} \longrightarrow \beta>0$ as $\quad n \rightarrow \infty$ and if $\mathbb{E}(w)=0$ and $n^{4} \mathbb{P}(|w| \geq n)=o(1)$, then $\lambda_{\max }\left(\Gamma_{n}\right)$ converges in probability to the nonrandom quantity, $(1+\sqrt{\beta})^{2} \mathbb{E}\left(w^{2}\right)$.

Recall, that the statement " $\lambda_{n}:=\lambda_{\max }\left(\Gamma_{n}\right)$ converges in probability to the limit $L:=$ $(1+\sqrt{\beta})^{2} \mathbb{E}\left(w^{2}\right)^{\prime}$ means that for each $\varepsilon>0$ one has

$$
\lim _{n \rightarrow \infty} \mathbb{P}\left(\left|\lambda_{n}-L\right| \geq \varepsilon\right)=0 .
$$

In particular this implies that for any $\delta>0$ there is a positive integer $N$, such that $n \geq N$ implies $\mathbb{P}\left(\lambda_{n} \geq L+\varepsilon\right)<\delta$.

In the present note we establish an estimate of the form $\mathbb{P}\left(\lambda_{\max }\left(\Gamma_{n}\right) \geq K\right) \geq \delta$, where $K \geq 1, \delta$ is small and dimensions of the matrix are large.

In the proposition below we use the following condition on the random variable $w$ :

$$
\forall t \geq 1, \alpha>0, c_{0}>0 \quad \mathbb{P}(|w| \geq t) \geq \frac{c_{0}}{t^{\alpha}} .
$$

Proposition 1. Let $\alpha \geq 2, c_{0}>0$. Denote by $X_{i}, 1 \leq i \leq p$ column-vectors of $W_{n}$. Let also $\left\{w_{i j}\right\}$ be i.i.d. random variables, identical copies of a certain random variable $w$, such that $\mathbb{E} w=0, \mathbb{E} w^{2}=1$ and satisfies condition (11). Then, for every $K \geq 1$,

$$
\mathbb{P}\left(\sup _{1 \leq i \leq p}\left|X_{i}\right| \geq \sqrt{K n}\right) \geq \min \left\{c_{0} p /\left(4 n^{\frac{\alpha}{2}-1} K^{\frac{\alpha}{2}}\right), \frac{1}{2}\right\} .
$$


In particular,

$$
\mathbb{P}\left(\lambda_{\max }\left(\Gamma_{n}\right) \geq K\right) \geq \min \left\{c_{0} p /\left(4 n^{\frac{\alpha}{2}-1} K^{\frac{\alpha}{2}}\right), \frac{1}{2}\right\}
$$

Remark 2. Note, that by Chebychev's inequality, $\mathbb{P}(|w| \geq t) \leq \frac{1}{t^{2}}$. Note also, that we use condition (1) in the proof only once, with $t=\sqrt{K n}$.

Remark 3. Note that if $\alpha<4$ and $p \geq \frac{2}{c_{0}} K^{\frac{\alpha}{2}} n^{\frac{\alpha}{2}-1}$, then, by condition (1), we have $\frac{n}{2} \mathbb{P}\left(w^{2} \geq K n\right) \geq \frac{n c_{0}}{2(K n)^{\frac{\alpha}{2}}}=\frac{c_{0}}{2(K)^{\frac{\alpha}{2}} n^{\frac{\alpha}{2}-1}} \geq \frac{1}{p}$. Therefore, one has $\mathbb{P}\left(\lambda_{\max }\left(\Gamma_{n}\right) \geq K\right) \geq \frac{1}{2}$ (see the Case 2 of the proof below).

Proof. For any $K \geq 0$,

$$
\begin{aligned}
\mathbb{P}\left(\sup _{1 \leq i \leq p}\left|X_{i}\right| \geq \sqrt{K n}\right) & =1-\mathbb{P}\left(\sup _{1 \leq i \leq p}\left|X_{i}\right| \geq \sqrt{K n}\right) \\
& =1-\mathbb{P}\left(\bigcap_{i=1}^{p}\left\{\left|X_{i}\right|<K\right\}\right) .
\end{aligned}
$$

But observe that for each $i,\left|X_{i}\right|$ depends on the entries of the $i$-th row of the matrix $W_{n}=\left(w_{i j}\right)$. Since the rows of $W_{n}$ are independent, it follows that $\left|X_{i}\right|$, for $i=1, \ldots, p$, are independent random variables. Also it follows that for each fixed $i$ we have that random vectors $\sum_{j=1}^{n} w_{i j}^{2}$ and $\sum_{j=1}^{n} w_{1 j}^{2}$ have the same distribution 1 and, with $w_{1}, \ldots, w_{n}$ independent copies of $w$, for each $i$ we have $\left|X_{i}\right| \sim\left|X_{1}\right| \sim \frac{1}{n} \sum_{j=1}^{n} w_{j}^{2}$. Therefore, continuing from (2)), we get

$$
\begin{aligned}
\mathbb{P}\left(\sup _{1 \leq i \leq p}\left|X_{i}\right| \geq \sqrt{K n}\right) & \geq 1-\prod_{i=1}^{p} \mathbb{P}\left(\left|X_{i}\right|<K\right) \\
& =1-\left(\mathbb{P}\left(\frac{1}{n} \sum_{j=1}^{n} w_{j}^{2}<K\right)\right)^{p}
\end{aligned}
$$

Now we will estimate the right hand side of (3) .

Observe that

$$
\mathbb{P}\left(\frac{1}{n} \sum_{j=1}^{n} w_{j}^{2} \geq K\right) \geq \mathbb{P}\left(\bigcup_{j=1}^{n}\left\{w_{j}^{2} \geq n K\right\}\right)
$$

\footnotetext{
${ }^{1}$ We write $x \sim y$ to say that $\mathrm{x}$ and $\mathrm{y}$ have the same distribution.
} 
For $j=1, \ldots, n$, consider the events $A_{j}=\left\{w_{j}^{2} \geq n K\right\}$. Since $w_{j}$ 's are i.i.d. with the same distribution as $w$, we have $\mathbb{P}\left(A_{j}\right)=\mathbb{P}\left(w^{2} \geq n K\right)$ for all $j$ and that events $A_{j}$ are independent. Using inclusion-exclusion principle, we have

$$
\begin{aligned}
\mathbb{P}\left(\bigcup_{j=1}^{n} A_{j}\right) & \geq \sum_{j=1}^{n} \mathbb{P}\left(A_{j}\right)-\sum_{j \neq k} \mathbb{P}\left(A_{j} \cap A_{k}\right) \\
& =\sum_{j=1}^{n} \mathbb{P}\left(w^{2} \geq n K\right)-\sum_{j \neq k}\left(\mathbb{P}\left(w^{2} \geq n K\right)\right)^{2} \\
& =n \mathbb{P}\left(w^{2} \geq n K\right)-\frac{n^{2}-n}{2}\left(\mathbb{P}\left(w^{2} \geq n K\right)\right)^{2} \\
& =\frac{n}{2} \mathbb{P}\left(w^{2} \geq n K\right)\left(2-(n-1) \mathbb{P}\left(w^{2} \geq n K\right)\right) .
\end{aligned}
$$

Using Chebychev's inequality $\mathbb{P}\left(w^{2} \geq n K\right) \leq \frac{1}{n K}$, we observe for $K \geq 1$

$$
2-(n-1) \mathbb{P}\left(w^{2} \geq n K\right) \geq 1
$$

Thus we obtain

$$
\begin{aligned}
\mathbb{P}\left(\lambda_{\max }\left(\Gamma_{n}\right) \geq K\right) & \geq 1-\left(1-\mathbb{P}\left(\frac{1}{n} \sum_{j=1}^{n} w_{j}^{2} \geq K\right)\right)^{p} \\
& \geq 1-\left(1-\frac{n}{2} \mathbb{P}\left(w^{2} \geq n K\right)\right)^{p}
\end{aligned}
$$

We consider two cases.

Case 1:

$$
\frac{n}{2} \mathbb{P}\left(w^{2} \geq K n\right) \leq \frac{1}{p} .
$$

In this case, using that $(1-x)^{p} \leq(1+p x)^{-1}$ on $[0,1]$, we get

$$
\mathbb{P}\left(\lambda_{\text {max }}\left(\Gamma_{n}\right) \geq K\right) \geq 1-\frac{1}{\frac{n p}{2} \mathbb{P}\left(w^{2} \geq K n\right)+1} .
$$

Using (6) our condition (11) with $t=\sqrt{K n}$, we get

$$
1 \geq \frac{n p}{2} \mathbb{P}\left(w^{2} \geq K n\right) \geq \frac{n p}{2} \frac{c_{0}}{(K n)^{\frac{\alpha}{2}}} .
$$

Thus,

$$
\mathbb{P}\left(\sup _{1 \leq i \leq p}\left|X_{i}\right| \geq \sqrt{K n}\right) \geq \frac{c_{0} p}{4 n^{\frac{\alpha}{2}-1} K^{\frac{\alpha}{2}}}
$$


Case 2:

$$
\frac{n}{2} \mathbb{P}\left(w^{2} \geq K n\right) \geq \frac{1}{p}
$$

In this case, using (10) and that $(1-x)^{p} \leq 1-\frac{p x}{2}$, on $\left[\frac{1}{p}, 1\right]$, we get

$$
\mathbb{P}\left(\sup _{1 \leq i \leq p}\left|X_{i}\right| \geq \sqrt{K n}\right) \geq \frac{n p}{4} \mathbb{P}\left(w^{2} \geq K n\right) \geq \frac{1}{2} .
$$

Now, combining (9) and (11) we obtain

$$
\mathbb{P}\left(\sup _{1 \leq i \leq p}\left|X_{i}\right| \geq \sqrt{K n}\right) \geq \min \left\{c_{0} p /\left(4 n^{\frac{\alpha}{2}-1} K^{\frac{\alpha}{2}}\right), \quad \frac{1}{2}\right\} .
$$

In particular part follows, since $\lambda_{\max }(\Gamma)=\left\|\Gamma_{n}\right\|=\frac{1}{n}\left\|W_{n}\right\|^{2} \geq \frac{1}{n} \sup _{1 \leq i \leq p}\left|X_{i}\right|^{2}$.

\section{References}

[1] J. Silverstein, On the weak limit of the largest eigenvalue of a large dimensional sample covariance matrix, J. of Multivariate Anal., 30 (1989), 2, 307-311.

[2] Y. Q. Yin, Z. D. Bai, P. R. Krishnaiah, On the limit of the largest eigenvalue of the large dimensional sample covariance matrix, Probab. Th. Rel. Fields., 78 (1988), 509-527. 